\title{
On the quantification of relativistic trajectories
}

\author{
Jean-Pascal Laedermann, PhD \\ independent researcher ${ }^{1}$
}

November 2021

1 pec@laedus.org 
Keywords

Abstract

From digitisation to quantification

Reminder on Schwarzschild, Kruskal and Szekeres

Appearance of tachyons

Transition from black hole to white hole

Cost of quantification

Refutability of the model

Two typical trajectories

Calculation tools

Analogy with quantum measurement

From the quantum to the infinitesimal

Bibliography 


\section{Keywords}

General relativity, geodesics, digitization, quantization, Schwarzschild, Kruskal-Szekeres, singularity, Lambert, tachyon, arbitrary precision calculation, complex time, Lorentz boost

\section{Abstract}

The geodesics on a relativistic manifold are given in a map $x$ by the well-known equation

$$
x^{\prime \prime \alpha}+\Gamma_{\mu \nu}^{\alpha} x^{\prime \mu} x^{\prime \mu}=0
$$

This equation can be solved numerically step by step and transposed into a quantization. We study here the effect of this quantization on the Schwarzschild spacetime, more precisely in the Kruskal-Szekeres map. 


\section{From digitization to quantification}

The geodesic equation is obtained by the Euler-Lagrange variational method, with the Lagrangian from the metric $g: L=g_{\mu \nu} x^{\prime \mu} x^{\prime \nu}$. The goal is to find the extremal solutions $\tau=$ $\int_{\lambda_{0}}^{\lambda_{1}} \sqrt{L} d \lambda$ which happens to be the eigentime of a test particle subjected to the field $g$. Except for the mass of the particle, which is in fact an energy, this proper time is an action.

Finding solutions digitally is extremely simple. We give ourselves a digitization step $\delta \lambda$ and an initial state $\left(x, x^{\prime}\right)$ of the mobile. The position $x$ is incremented by $x^{\prime} \delta \lambda$. The geodesic equation gives $x^{\prime \prime} \alpha=-\Gamma_{\mu \nu}^{\alpha} x^{\prime} \mu x^{\prime}$ and the velocity $x^{\prime}$ is incremented by $x^{\prime \prime} \delta \lambda$. Then the process is iterated.

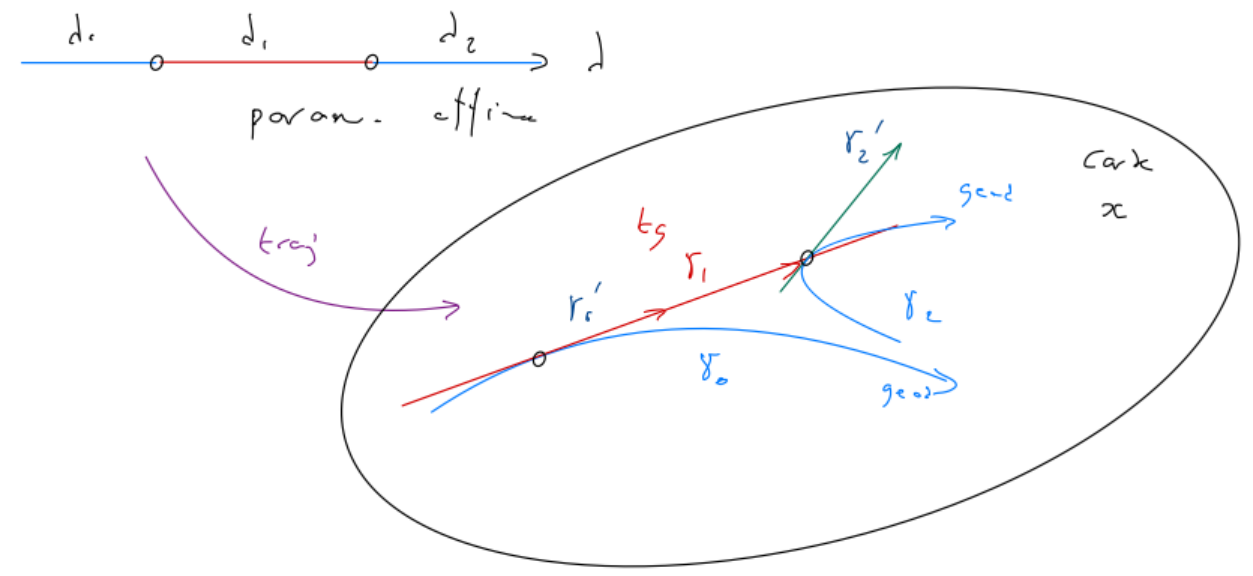

Fig 1: A mobile moves from a geodesic $\gamma_{0}\left(\lambda_{0}\right)$ to a geodesic $\gamma_{2}\left(\lambda_{2}\right)$ by a trajectory element $\gamma_{1}\left(\lambda_{1}\right)$ on an interval $\delta \lambda$ This is a straight line in the tangent space. We use the fact that the tangent spaces $T_{x} \mathbb{R}^{n}$ are in fact canonically included in $\mathbb{R}^{n}$.

The choice of the affine step will be made here by keeping the time step constant $ð \tau$ which gives

$$
\delta \lambda=ð \tau / \sqrt{L}
$$

This time step can be physically equated with a quantum of action in the following interpretation.

At each step, the mobile requests a quantum according to the chosen coordinate system $x$. It uses this quantum to continue its trajectory in its local context, which is the tangent space to the space-time manifold at the current point. Then the new state is considered as such on the global space-time. An observer placed on the particle moves during the quantum of time according to a trajectory linearized by the choice of its map.

Some remarkable facts emerge.

Firstly, the coordinate system chosen by the observer is essential. The linearization of the trajectory during $ð \tau$ depends on the map $x$ and makes the interaction between space-time and the observer contextual. There is an effect of the observation on the trajectory.

Secondly, it cannot be excluded that the quantization step involves speeds higher than that of light. This phenomenon can be related to certain quantum effects, such as the possibility for a 
particle to tunnel through a potential barrier, or to violate the conservation of energy law for a time short enough to be allowed by Heisenberg's uncertainty relations.

Third, in the particular case of the Schwarzschild model with a radius $r_{S}$ it becomes possible to be in the forbidden zone beyond the bare singularity described below.

\section{Reminder on Schwarzschild, Kruskal and Szekeres}

In 1916, Karl Schwarzschild was one of the first to find a solution to the gravitational equations of Einstein's general relativity. This solution, which describes the field created by a point mass, is expressed by the following metric in polar coordinates, with a speed of light $c=1$ and a Schwarzschild radius $r_{S}$ :

$$
d \tau^{2}=\left(1-\frac{r_{S}}{r}\right) d t^{2}-\left(1-\frac{r_{S}}{r}\right)^{-1} d r^{2}-r^{2}\left(d \theta^{2}+\sin ^{2} \theta d \varphi^{2}\right)
$$

Two peculiar rays are immediately noticeable. The first one, $r=r_{S}$ gives the horizon beyond which a particle cannot escape, giving the name of black hole to this zone. The second one, $r=$ 0 , is a singularity of the metric, known as bare, where any particle entering the black hole ends its trajectory in a finite time.

The Kruskal-Szekeres coordinate transformation leads to a formulation in terms of the variables $(T, X, \theta, \varphi)[1]$ :

$$
d \tau^{2}=\frac{4 r_{s}^{3}}{r} e^{-\frac{r}{r_{S}}}\left(d T^{2}-d X^{2}\right)-r^{2}\left(d \theta^{2}+\sin ^{2} \theta d \varphi^{2}\right)
$$

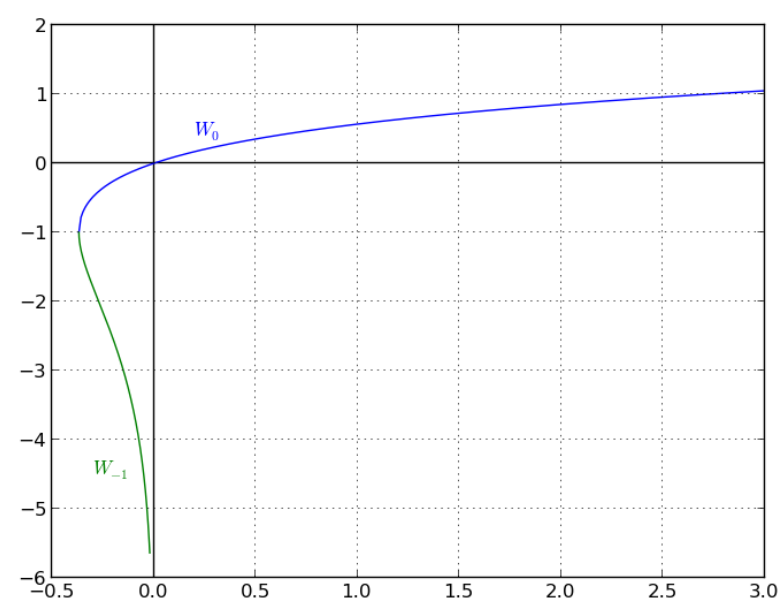

The parameter $r=r_{S}\left(\mathcal{W}_{0}\left(\frac{X^{2}-T^{2}}{e}\right)+1\right)$ is given by the branch 0 of the Lambert function $\mathcal{W}$ 


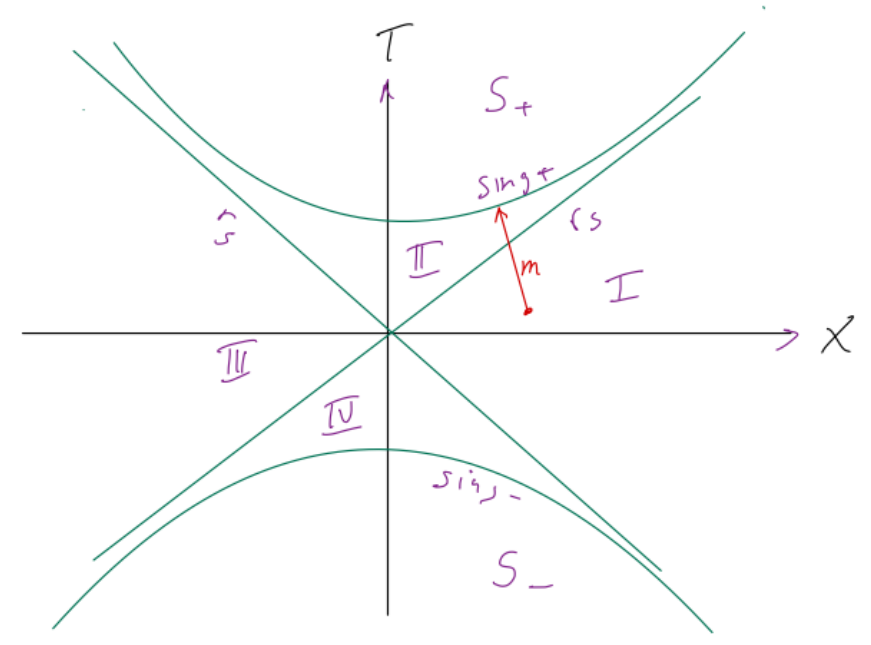

Fig 2: Real branches of the Lambert function

Fig 3. Kruskal-Szekeres map

The diagram in Fig 3 shows the following regions:

I space-time outside the black hole

II black hole

III another related component of space-time

IV white hole

$\mathrm{S}_{+} \quad$ inside of the bare singularity

S- other component of the interior of the bare singularity

This map shows that the Schwarzschild horizon is not a physical singularity, but only an artefact due to the choice of the map.

The diagonal lines represent the Schwarzschild horizon, and the two boundary branches of the sing+ and sing- hyperbola the entrance and exit of the bare singularity itself.

A particle from region II ends its trajectory on sing+, without being able to get out. Conversely, a particle in region IV cannot do anything else but exit, hence the name white hole. We also find the expressions sink and source for these two regions.

S- and S+ are inaccessible, or forbidden, because they are outside the map domain. These two regions and their boundaries are associated with a single point, the zero of the polar coordinates, and can be considered as collapsed.

At least in the hypothesis of a strictly continuous world... 


\section{Appearance of tachyons}

Traditionally, the term tachyon is applied to a hypothetical particle with a speed greater than the speed of light. The exit of the speed of the cone of the future is translated by the fact that $L<0$ and thus an imaginary quantization step. It is proposed here to use a complex proper time:

$$
\tau=\tau_{r}+i \tau_{i} \in \mathbb{C}
$$

This time is measured by two clocks, one real and the other imaginary. The increase of the affine parameter becomes $\delta \lambda=ð \tau_{r} / \sqrt{L}$ if $L>0$ or $\delta \lambda=i ð \tau_{i} / \sqrt{-L}$ if $L<0$. In this way, the trajectory remains real in the map $x$. For a tachyon, it is the imaginary clock that works, the other one remaining fixed, and the opposite for a standard particle.

For any coordinate system on space-time, the notions of time and space are found locally by placing in the tangent space an orthonormal basis which diagonalizes the metric, then, thanks to a possible permutation of the axes and a calibration of the units, to obtain the diagonal Minkowski matrix $\operatorname{Diag}(1,-1,-1,-1)$. The 0 coordinate is then time and the others define space. The base obtained in this way is called a tetrad.

The proper speed $\dot{x}=\frac{\delta \lambda}{\partial \tau} x^{\prime}$ is transformed into a quad speed $\boldsymbol{u}=\gamma\left(\begin{array}{l}1 \\ \boldsymbol{v}\end{array}\right)$ where $\boldsymbol{v}$ is the space velocity of the mobile. Let $v$ be its Euclidean norm and $\boldsymbol{n}_{G}$ the unit vector $\boldsymbol{v} / v$ unit vector, the socalled slip vector. We easily obtain $\gamma=\left(1-v^{2}\right)^{-1 / 2}$.

If $v<1$, it is possible to put the mobile at rest with a Lorentz boost $\Lambda(v)$ i.e.

$$
\Lambda(\boldsymbol{v}) \boldsymbol{u}=\left(\begin{array}{l}
1 \\
\mathbf{0}
\end{array}\right)
$$

If $v>1, \gamma$ becomes pure imaginary. Nevertheless, it is possible to extend the boost by

$$
\Lambda(\boldsymbol{v})=\Lambda\left(\frac{\boldsymbol{n}_{G}}{v}\right) R\left(\boldsymbol{n}_{G}, \frac{\pi}{2}\right)
$$

where $R(\boldsymbol{n}, \theta)$ is a rotation of angle $\theta$ and axis $\boldsymbol{n}$. It can be seen that $\Lambda(\boldsymbol{v}) \boldsymbol{u}=\left(\begin{array}{c}0 \\ \boldsymbol{i n}_{G}\end{array}\right)$. The "resting" with this extended boost makes a particle appear in the direction $\boldsymbol{n}_{G}$ with a proper time marked by its imaginary clock. As $1 / v<1$, this transformation is physically feasible for an external observer, and the tachyon could be visible. One can notice that the factor $\boldsymbol{i}$ in front of $\boldsymbol{n}_{G}$ is consistent, since it does imply a quadrivector of unit Minkowskian norm. 


\section{Transition from black hole to white hole}

The appearance of a state in the zone forbidden by the singularity poses a more delicate problem. Indeed, the Christoffel coefficients involve the parameter

$$
r=r_{s}\left(\mathcal{W}_{0}\left(\frac{1}{e}\left(X^{2}-T^{2}\right)\right)+1\right)
$$

This critical zone is defined by $X^{2}-T^{2}<-1$ which takes the domain of $\mathcal{W}_{0}$.

The solution proposed here is to use the other part of this function on the real line, namely

$$
r=r_{s}\left(\mathcal{W}_{-1}\left(\frac{1}{e}\left(X^{2}-T^{2}\right)^{-1}\right)+1\right)
$$

by reversing the term $X^{2}-T^{2}$ which is in the domain of $\mathcal{W}_{-1}$. The continuation of the trajectory is then done by changing the signs of $T$ and $X$, which turns the mobile from a black hole to a white hole. This idea is suggested by the hyperbolic character of the Kruskal map.

\section{Cost of quantification}

The evolution of the trajectory during the time quantum is no longer geodesic, and therefore requires some work. The force that appears during this displacement is given by

$$
f^{\alpha}=x^{\prime \prime \alpha}+\Gamma_{\mu \nu}^{\alpha} x^{\prime} \mu x^{\prime v}
$$

and its work on the affine segment $\delta \lambda$ is given by

A quick calculation shows that

$$
\delta W=\int_{\lambda_{0}}^{\lambda_{1}} g_{\mu \nu} f^{\mu} x^{\prime \nu} d \lambda
$$

$$
\delta W=\frac{1}{2} \delta \lambda x^{\prime \mu} x^{\prime v} x^{\prime \rho} \int_{0}^{1} \frac{\partial g_{\mu \nu}}{\partial x^{\rho}}\left(x+x^{\prime} \xi \delta \lambda\right) d \xi=\delta \lambda x^{\prime \mu} x^{\prime v} x^{\prime \rho} \int_{0}^{1} \Gamma_{\rho \mu \nu}\left(x+x^{\prime} \xi \delta \lambda\right) d \xi
$$

This expression makes it possible to estimate the energy needed to quantify the movement. 


\section{Refutability of the model}

Given a time quantum, one can ask what mass $M_{0}$ corresponds to a quantum of action equal to Planck's constant. In other words $M_{0} c^{2} \delta \tau=\hbar$.

It is clear that the finer the digitization, the closer the trajectories are to the unquantised geodesics, thus delaying the onset of the quantum effects mentioned above.

The smaller the quantum, the later the effect, and the longer the calculation time. The calculations carried out here allow us to aim for a time quantum of about $10^{-13} \mathrm{~s}$ which corresponds to a mass of $10^{-2} \mathrm{eV} / \mathrm{c}^{2}$. To reach the mass of the neutrino, for example, which is currently estimated at $1.1 \mathrm{eV} / \mathrm{c}^{2}$, would need a temporal resolution two orders of magnitude lower, resulting in calculation times that are about 100 times longer. As the calculations performed here require several days, it is not impossible to think that an optimization could be achieved up to the level of actually observable particles.

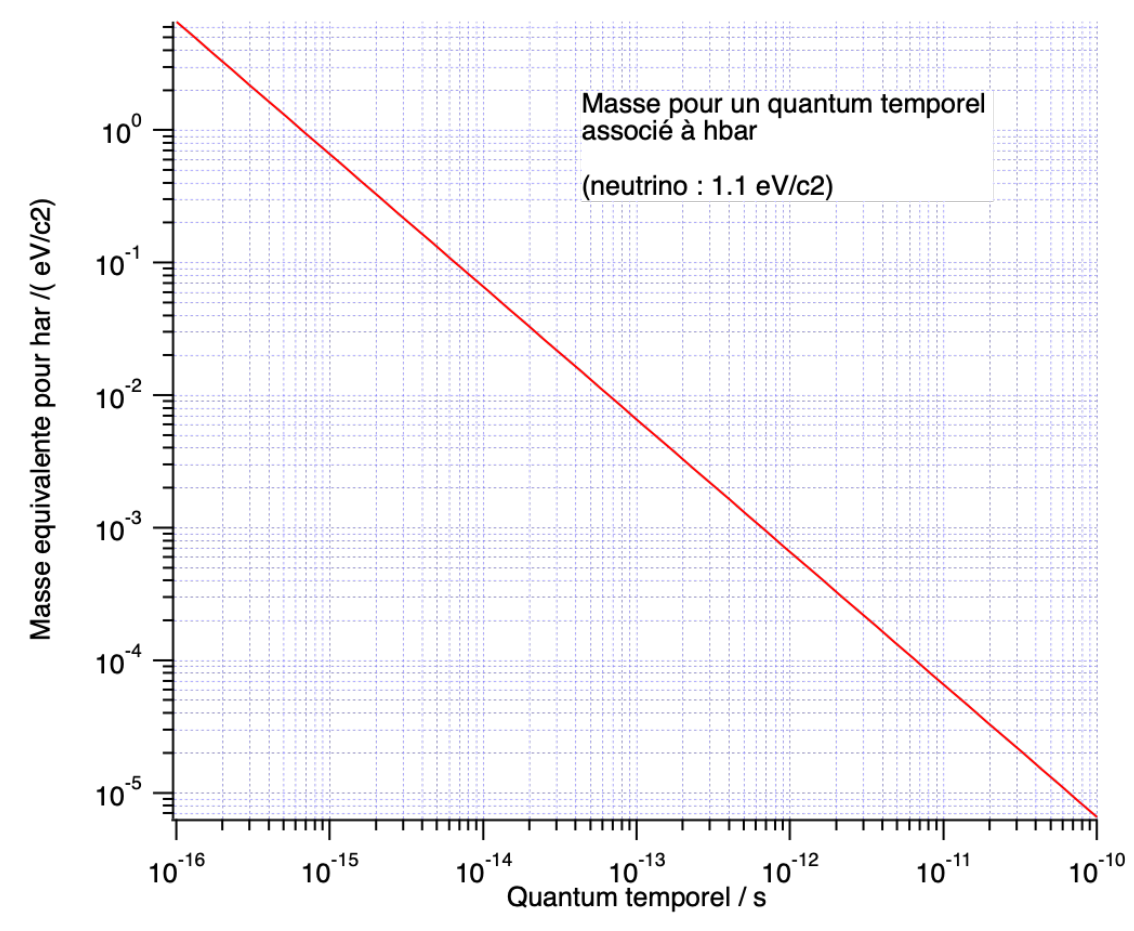

Fig 4. Mass - time quantum relationship 


\section{Two typical trajectories}

In general, the trajectories end either with the limiting velocity 1 or at the singularity. Tachyons are fleeting, and return to standard space-time with a final velocity of 1 .
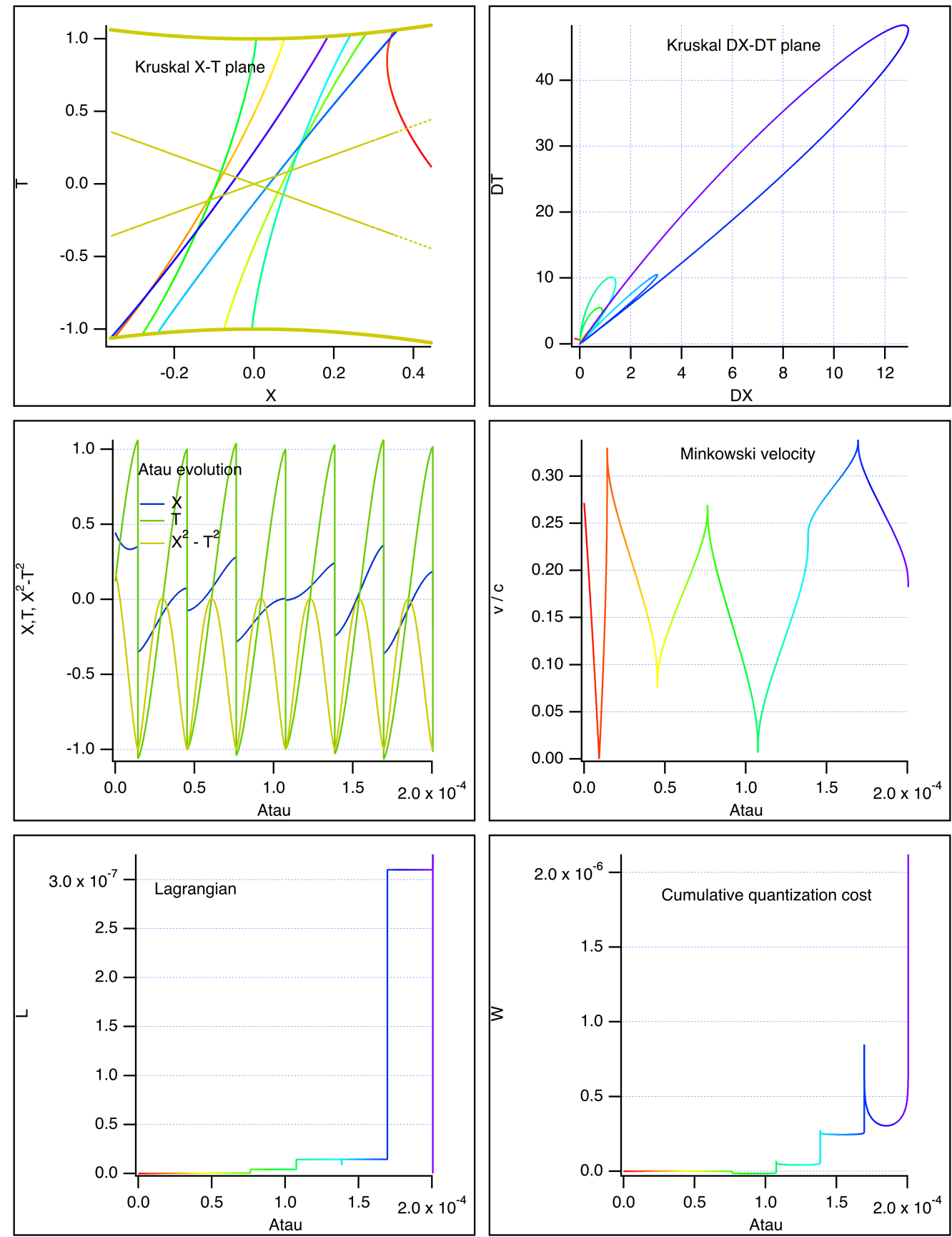

Fig 5. Trajectory evolving towards the singularity. The variable Atau is simply the addition of the two real and imaginary clocks. The imaginary time is identified by a negative Lagrangian. The start of the trajectory is in red and its end in dark blue. The passages through the singularity are located at the points where $X^{2}-T^{2}<-1$ 

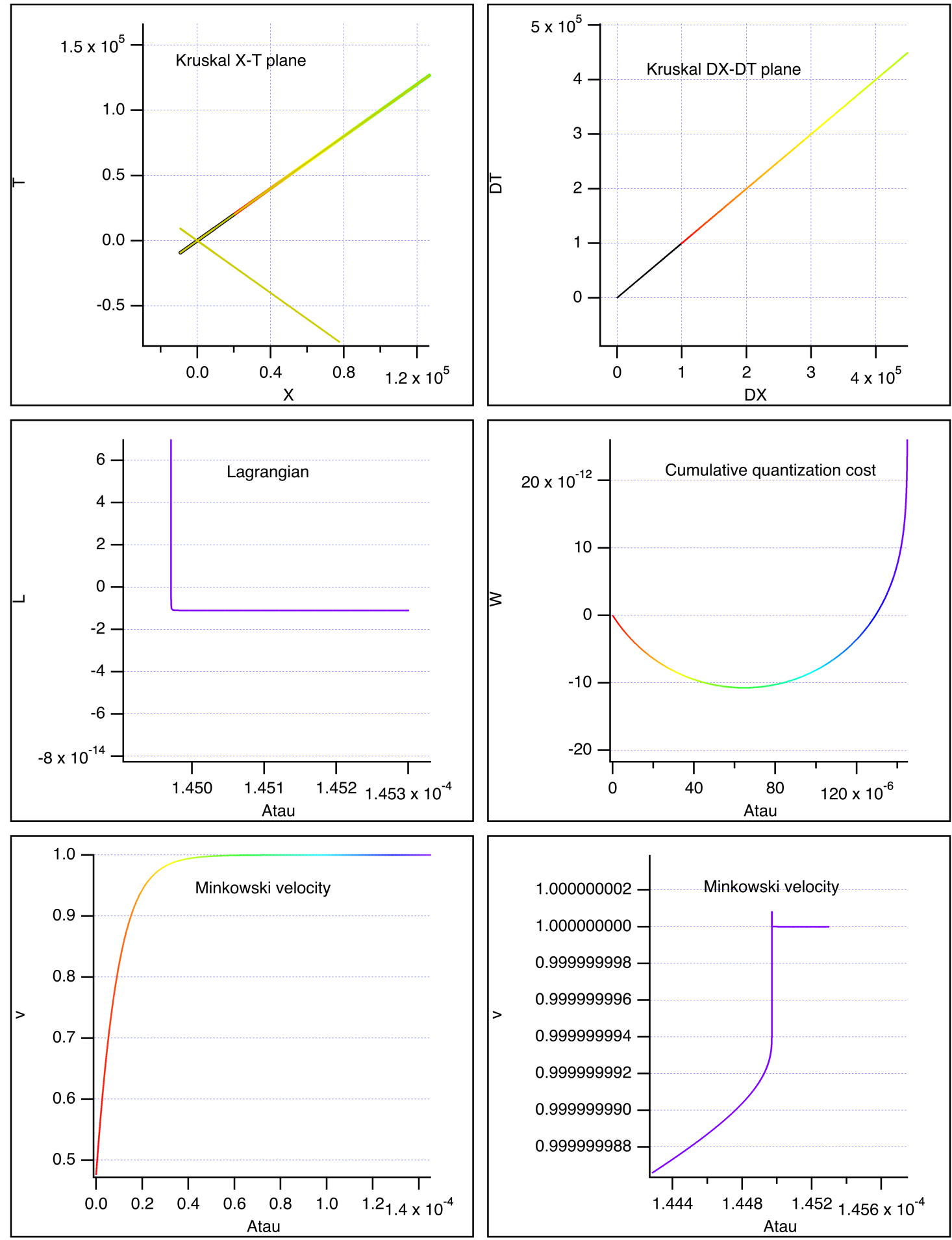

Fig 6. Trajectory leading to a tachyon, before ending on the singularity. The colouring of the top two graphs is given by the imaginary clock from the black part. The calculation was redone by increasing the precision from 4096 to 8192 bits, with no significant difference. 


\section{Calculation tools}

The digital tracking of trajectories is done over several million steps. The standard precision of current computers (double precision) is 53 bits, which is totally insufficient. The MPFR library [3] implements the calculation with an arbitrary precision, only limited by the machine's memory. An interface written by P. Holoborodko [3] then allows the use of the Eigen vector calculation library [4]. The very complete study of $F$. Johansson [5] on the Lambert function finally makes it possible to carry out the calculation of trajectories, which becomes stable with a precision of 4096 bits (approximately 1200 decimal places).

The exploration of the various trajectories is programmed in $C++$ and uses a 128 processor machine running in the Gnu - Linux Ubuntu 20.4 environment.

The trajectories presented here generally require several days of parallel CPU.

\section{Analogy with quantum measurement}

Some of the effects of observing a relativistic motion during a time quantum ð $\tau$ have been described above. In summary, the motion naturally follows a geodesic, then during the time of observation, it follows a tangent, then it resumes its natural trajectory, but on another, neighbouring geodesic.

This sequence is similar to the Copenhagen version of quantum measurement, where there are two possible evolutions for a quantum system. The first, known as unitary (U-type), is governed by the Schroedinger or Dirac equation. The second, which appears when the system is measured, is called wave packet reduction (type $R$ ), and consists of projecting this wave function onto an eigen space associated with the observable to be measured.

Let $A$ be the self-adjoint operator translating an observable $A$. To measure $A$ following the Geneva school [8], is to ask a series of questions whose answers are yes or no. A question about $A$ is for example: "Will the value of $A$ appear in a certain interval $\Delta$ of the real line".

Let $S p A$ be the spectrum of the operator $A$. This question is represented by the projection operator $J_{\Delta}=\sum_{a \in \Delta \cap S p A} J_{a}^{A}$ where $J_{a}^{A}$ is the projector onto the eigenspace of eigenvalue $a^{2}$. The result of the measurement, i.e. the answer to the question, will be yes with probability $p_{1}=$ $\left\langle\psi\left|J_{\Delta}\right| \psi\right\rangle$ and the system will then be in the state $|1\rangle=\frac{J_{\Delta} \psi}{\left\|J_{\Delta} \psi\right\|}$. The answer no is treated in the same way, but with the projector $J_{C \Delta}$ and gives the final state $|0\rangle$.

One can imagine that the measurement lasts for a time interval $ð \tau$ and, after the response has been randomly chosen, the wave function evolves "linearly" towards its final state.

$$
{ }^{2} A=\sum_{a \in S p A} a J_{a}^{A}
$$

The continuous version involves an integral, which does not change the discussion. 


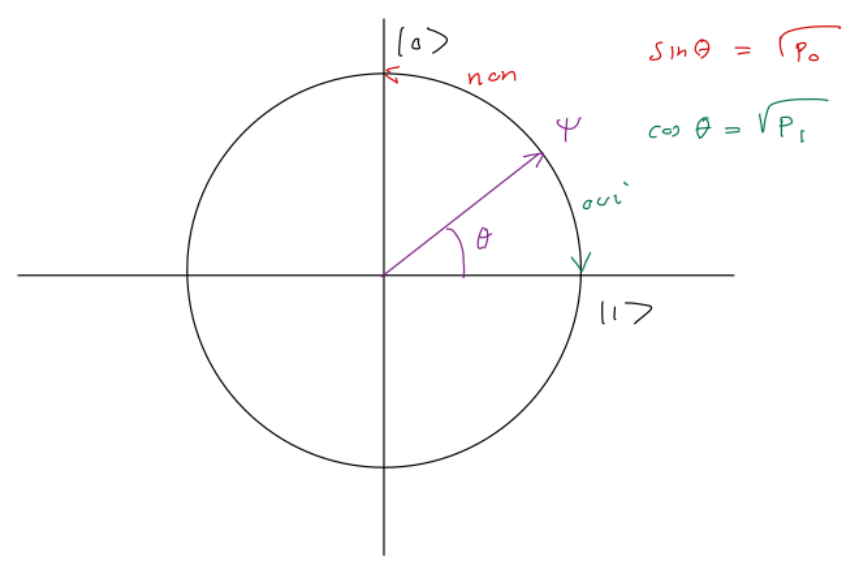

Fig 7. Evolution of the quantum probability amplitude in $R$ mode

For example, the path in Fig 7 :

$$
t \mapsto \psi_{t}=\cos \left(\theta\left(1-\frac{t}{\partial \tau}\right)\right)|1\rangle+\sin \left(\theta\left(1-\frac{t}{\partial \tau}\right)\right)|0\rangle \cos \theta=\sqrt{p_{1}}
$$

moves in a uniform and unitary manner from $\psi$ à $|1\rangle$ in case of a yes answer.

For the Schroedinger equation, this evolution is governed in the $(|1\rangle,|0\rangle)$ basis by the Hamiltonian operator

$$
H_{1}=\frac{\theta \hbar}{\partial \tau} \sigma_{2} \theta=\arccos \sqrt{p_{1}}
$$

where $\sigma_{2}$ is the second Pauli matrix. It can be seen that $\left\langle H_{1}\right\rangle=0$

$$
\psi_{t}=e^{\frac{\theta t}{\partial \grave{\partial} \tau} \sigma_{2}} \psi=e^{\frac{\theta t}{\partial \tau}(|1\rangle\langle 0|-| 0\rangle\langle 1|)} \psi
$$

Initially, the wave function follows a trajectory $U$ given by a Hamiltonian $H$. During the measurement, the reduction $R$ is replaced by a trajectory $U$ with a Hamiltonian proportional to $\sigma_{2}$.

It then resumes the trajectory $U$ given by $H$.

\section{From the quantum to the infinitesimal}

The infinitesimals of Leibnitz and Newton have only recently been given a consistent axiomatic basis. They have been used systematically by mathematicians like Euler, Lagrange or Wallis with success and without rigorous justification. Physicists use them without further ado on a daily basis. The axiomatization of continuity by $d$ 'Alembert, Cauchy and Weierstrass almost sounded the death knell of these quantities, as small as one likes, but non-zero...

Nevertheless, they have made a surprising reappearance through topos, equipped with their not necessarily Boolean logics. For smooth infinitesimal analysis, for example, they are defined by the subset of the line $\Delta=\left\{\varepsilon \mid \varepsilon^{2}=0\right\}$ which is no longer reduced to $\{0\}$. One then speaks of nilpotent real numbers. This has the effect of eliminating all powers greater than or equal to 2 in the Taylor developments on this set. In other words, any function becomes linear on $\Delta$ or : $\Delta$ is a representation of the tangent space in zero which is included in the real line. 
The above analysis performs this integration with the idea that the time quantum could ideally be understood from the nilpotents.

The quantum effects in the vicinity of singularities are reminiscent of John Lane Bell's formula:

$$
\text { Vale } i c t \text {, ave } i \varepsilon ![6]^{3}
$$

as an extension of the discussion on the introduction of imaginary time by Minkowski [7].

J.-P. Laedermann, November 2021

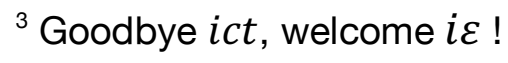




\section{Bibliography}

[1] T. Müller

[2] P. Holoborodko

[3] Gnu

[4] Eigen

[5] F. Johansson

Computing the Lambert $W$ function in arbitrary-precision complex interval arithmetic.

https://arxiv.org/abs/1705.03266

[6] J. L. Bell A Primer of Infinitesimal Analysis, 2nd Edition,

[7] W. Misner, S. Thorne, J.-A. Wheeler

[8] C. Piron
Catalogue of Spacetimes, 2.2.5,

https://arxiv.org/abs/0904.4184v3

http://www.holoborodko.com/pavel/mpfr/ https://www.mpfr.org/

https://gitlab.com/libeigen/eigen
Cambridge 2008, p80

Gravitation, Box 2.1 p51

Princeton University Press 2017

Quantum Mechanics, PPUR 1990 